\title{
A Study of Artificial Sweeteners and Thyroid Cancer Risk
}

\author{
Navdeep Singh ${ }^{\mathrm{a}, \mathrm{e}}$, Sandeep Singh Lubana ${ }^{\mathrm{b}}$, Saurabh Arora ${ }^{\mathrm{c}}$, Issac Sachmechi ${ }^{\mathrm{d}}$
}

\begin{abstract}
Background: In recent decades, data from certain observational studies have stirred controversy over artificial sweeteners by linking them with certain malignancies. As the incidences of artificial sweetener consumption and thyroid cancer are both increasing, our study aimed to determine any possible association between them.
\end{abstract}

Methods: This retrospective observational study enrolled 50 patients (group 1) with proven diagnosis of well-differentiated thyroid cancer (WDTC) and 50 control subjects (group 2) diagnosed as having benign thyroid nodule by fine-needle aspiration. The survey questionnaire included the total amount and duration of intake of artificial sweeteners.

Results: Increased consumption of artificial sweeteners was noted in group 1 as compared to group 2, which was statistically significant ( $76 \%$ vs. $24 \%, \mathrm{P}<0.01)$. This study suggested that the use of an average of four packets $(4 \mathrm{~g})$ per day of artificial sweetener for an average duration of 5 years is associated with WDTC.

Conclusions: Our study emphasizes the significance of artificial sweetener consumption as a potential risk factor for WDTC and increase in public awareness regarding this association if other studies in future report similar findings.

Keywords: Artificial sweeteners; Well-differentiated thyroid cancer; Benign thyroid nodule

\section{Introduction}

Artificial sweeteners, also known as non-nutrient sweeteners,

Manuscript submitted June 8, 2020, accepted June 17, 2020

Published online July 1, 2020

aDepartment of Internal Medicine, North Shore University Hospital, Manhasset, NY 11030, USA

bDepartment of Hematology/Oncology, State University of New York (SUNY) Downstate Medical School, 450 Clarkson Ave., Brooklyn, NY 11203, USA

'Department of Endocrinology, Dayanand Medical College and Hospital, Tagore Nagar, Ludhiana, Punjab 141001, India

dDepartment of Endocrinology, Icahn School of Medicine at Mount Sinai/ Queens Hospital Center, 82-68 164th St., Jamaica, NY 11432, USA

${ }^{\mathrm{e} C}$ Corresponding Author: Navdeep Singh, Department of Internal Medicine, North Shore University Hospital, 300 Community Dr., Manhasset, NY 11030, USA. Email: imedsingh@gmail.com

doi: https://doi.org/10.14740/jocmr4258 are substances used as additives to food and beverages as a replacement for sugar. Saccharin was the first artificial sweetener discovered accidentally in 1879 by coal scientist, who forgot to wash his hands before the meal, which caused the bread to taste unusually sweet [1]. The use of saccharin gained popularity during World War I and II when sugar production declined due to the agricultural crisis. The economic expansion following World War II marked an era of rising living standards in western countries, and the ground to use artificial sweeteners shifted from expenditure to calorie reduction [2]. However, a higher concentration of saccharin was associated with characteristic bitter and metallic aftertaste in some people [3], which was found to increase as the concentration of this artificial sweetener rises and was responsible for flattening of sweetness function [4].This hurdle was overcome in 1950s when researchers found that the blending of saccharin and cyclamate reduced the bitter aftertaste caused by saccharin. With the recognition of the deleterious effect of sugar on human health in recent years on the background of the rising pandemic of obesity, the consumption of artificial sweeteners has increased dramatically in the USA [5]. The artificial sweeteners are arbitrarily classified into first-generation and second-generation sweeteners. The first-generation compounds include saccharin, cyclamate, and aspartame. Acesulfame K, alitame, sucralose, and neotame were referred to as new or second-generation artificial sweeteners. However, even secondgeneration sweeteners share similar drawbacks, as seen with the first-generation compounds [6]. The sweet taste is often coupled with the metallic aftertaste and does not provide the desirable flavor of regular sugar. The combination of various available sweeteners has led to an improvement in the taste of sweetened food items.

Researchers have debated the safety of artificial sweeteners for a long time. The first big blow to the artificial sweetener industry was observed in 1970 when the Food and Drug Administration (FDA) banned cyclamate from all dietary foods and beverages in the USA [1]. The FDA ban was based on the experimental data that showed an elevated risk of bladder carcinoma in rodents fed on high doses of cyclamate and saccharin. However, subsequent studies on human beings failed to replicate the experimental findings [7]. Another study published by Sturgeon et al [8] suggested an increased risk of bladder carcinoma with heavy consumption ( $>1,680 \mathrm{mg}$ per day) of artificial sweetener. A study conducted by Lim et al [9] including 285,079 men and 188,905 women aged 50 to 71 years over a period of 5 years, did not find any association between aspartame intake and risk of hematopoietic or neurological malignancies. One prospective study showed a link between non-nutrient sweetener aspartame intake and development of 
lymphoma and leukemia only in men [10]. The question of whether or not artificial sweetener consumption is associated with an increased risk of cancer is currently a source of both investigation and controversy.

There is a dearth of literature on the potential role of artificial sweeteners in thyroid carcinogenesis. The National Cancer Institute's Surveillance, Epidemiology, and End Results (SEER) program has reported an increase in the prevalence of WDTC [11]. In the USA, the average yearly rise in thyroid carcinoma incidence rate was 5\% over the last decade [12]. Currently, thyroid cancer is the fifth most commonly detected carcinoma in women in the USA. The factors responsible for the rising incidence of thyroid carcinoma have been highly debated. This increase is partially attributed to increased diagnostic scrutiny [13]. However, other unknown environmental and dietary factors are likely to contribute to a significant proportion of thyroid cancer risk. To the best of our knowledge, no study has been conducted to determine the association of artificial sweetener consumption with thyroid carcinoma. As the incidences of artificial sweetener consumption and thyroid cancer are increasing, our study aimed at determining a possible association between the two.

\section{Materials and Methods}

After approval by the Ethical Review Boards, this study was conducted in accordance with the Declaration of Helsinki. The purpose and methodology of the study was explained to the study participants and signed informed consent was obtained from each participant.

A retrospective analysis of the medical records of a series of patients from January 1, 2004 to August 31, 2014 with a history of thyroid nodule was conducted in two city hospitals; Icahn School of Medicine at Mount Sinai/Queen Hospital Center and Elmhurst Hospital Center. All patients were aged 18 years and above. We found 100 patients for the study; these 100 patients were divided into two different groups. Seventysix $(76 \%)$ patients were female, twenty-four $(24 \%)$ were male with a male to female ratio of $1: 3$. The mean age of the patients was 53.6 years.

The study group (group 1) comprised of 50 patients who underwent total thyroidectomy for preoperative diagnosis of well-differentiated thyroid cancer (WDTC); the control group (group 2) consisted of 50 patients who were diagnosed to have benign thyroid nodules via fine-needle aspiration biopsy (FNAB).

A telephonic survey was conducted in both groups. The survey questionnaire was pertaining to the use of artificial sweeteners in their diet. The questionnaire included the total amount and duration of artificial sweetener consumed as tabletop sweetener and beverages by the subjects. The duration for which the artificial sweetener was consumed was based on the history given by the patients. Consumption of artificial sweetener in the form of snacks/ice creams was not taken into account as these are not consumed on routine basis and hence it was unlikely to affect the outcome. The questionnaire did not specifically include the details on the individual type of artificial sweetener used because it was not possible to study the effect of individual sweetener on the outcome as mixing of two or more sweeteners is commonly done.

The amount of ingredients acesulfame potassium, aspartame, saccharin and sucralose in one packet $(1 \mathrm{~g})$ of tabletop artificial sweetener was derived from the published data on the use of high-intensity artificial sweeteners in food by US FDA [14]. Each 1-g packet of tabletop artificial sweetener contains either $39 \mathrm{mg}$ of acesulfame potassium (Sunett ${ }^{\circledR}$ and Sweet One ${ }^{\circledR}$ ) or $40 \mathrm{mg}$ of aspartame (Nutrasweet ${ }^{\circledR}$, Equal ${ }^{\circledR}$, and Sugar Twin ${ }^{\circledR}$ ) or $20 \mathrm{mg}$ saccharin (Sweet and Low ${ }^{\circledR}$, Sweet Twin $^{\circledR}$, Sweet'N Low ${ }^{\circledR}$, and Necta Sweet ${ }^{\circledR}$ )or 13 mg of sucralose $\left(\right.$ Splenda $\left.^{\circledR}\right)$. Depending upon the amount and type of ingredients (acesulfame potassium, aspartame, saccharin and sucralose) [15] we calculated the number of packets each different type of beverage contains as shown in Table 1. For example, one can of Diet Coke contains $125 \mathrm{mg}$ of aspartame and one packet of tabletop sweetener contains $40 \mathrm{mg}$ of aspartame. Hence a can of Diet Coke would contain 3.125 packets $(3.125$ g) of tabletop artificial sweeteners.

Data regarding demographics, history of head and neck radiation exposure in childhood and history of first degree relative with thyroid cancer was also collected. Statistical analysis was performed by Chi-square test, with Yates' correction where appropriate, a $\mathrm{P}$ value of less than 0.05 indicated statistical significance. Binary logistic regression analysis was performed to estimate the odds ratio (OR) and 95\% confidence interval (CI).

\section{Results}

\section{Group 1}

Out of the 50 patients enrolled in group 1, and 40 (80\%) were women with male to female ratio of $1 / 4$. The mean age at diagnosis was 52.4 years (range: 28 - 74 years). The mean body mass index in group 1 was $26.45 \pm 3.96 \mathrm{~kg} / \mathrm{m}^{2}$. Papillary thyroid carcinoma was confirmed at histopathological examination in $42(84 \%)$ patients, while follicular thyroid carcinoma was the final diagnosis in eight patients $(16 \%)$. Three $(6 \%)$ patients in this group declared a family history of thyroid malignancy in first-degree relatives, and one $(2 \%)$ patient had a history of head and neck irradiation in childhood. Thyroid function tests were normal in $44(88 \%)$ patients in this group, while six $(12 \%)$ patients had subclinical hypothyroidism at the time of presentation.

\section{Group 2}

Seventy-two percent of patients enrolled in this group were females (36 patients) with a male to female ratio of $1 / 2.6$; the mean age was 54.6 years (range: 31 - 78 years). The mean body mass index in group 2 was $27.34 \pm 4.52 \mathrm{~kg} / \mathrm{m}^{2}$. The diagnosis of benign thyroid nodular disease was confirmed by histological examination in all the patients. One $(2 \%)$ patient in this group declared a family history of thyroid disease in the 


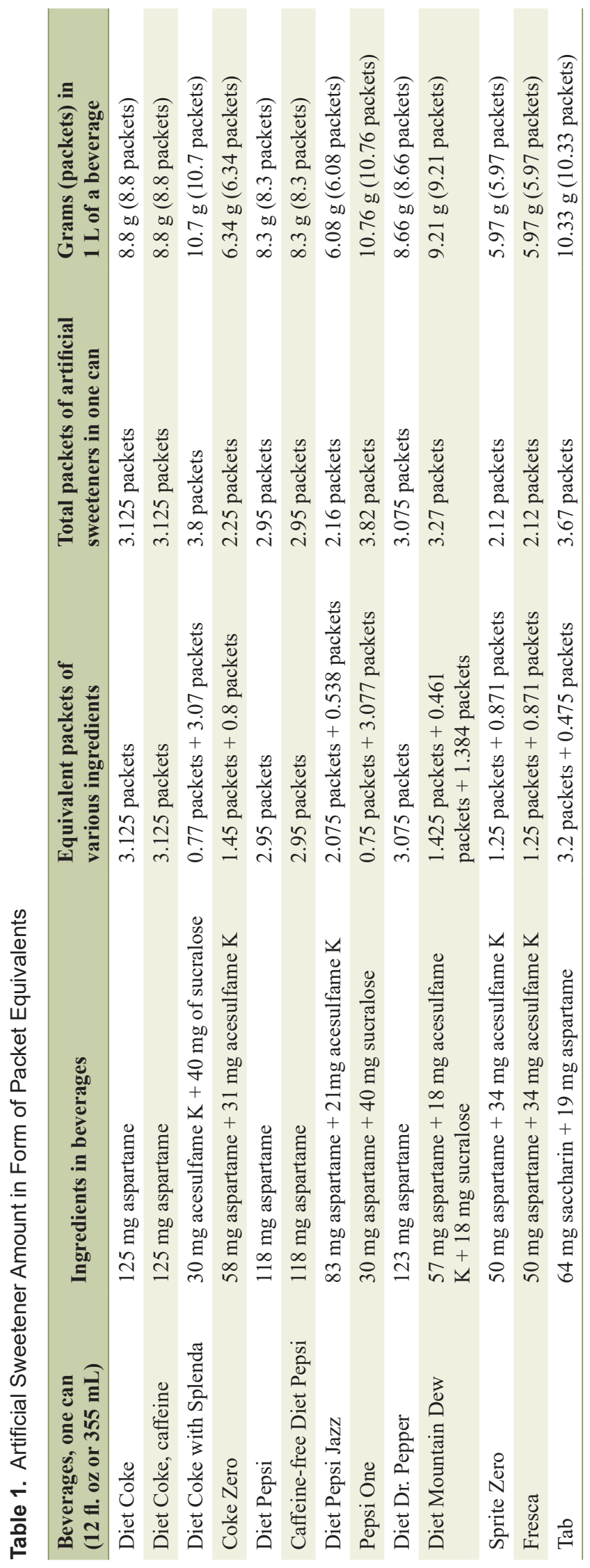

first-degree relative, while none of the patients in this group had a history of head and neck irradiation in childhood. Out of 50 patients, $40(80 \%)$ were biochemically euthyroid at the time of presentation while eight (16\%) patients had subclinical hypothyroidism, and two (4\%) patients were diagnosed with overt hypothyroidism.

History of artificial sweetener consumption was noted in 38 out of 50 patients $(76 \%)$ in group 1, while in group 2, only 12 out of 50 patients $(24 \%)$ consumed artificial sweetener. The average amount of artificial sweetener consumption in group 1 was $4 \mathrm{~g}$ (four packets) per day for an average duration of 5 years. In group 2, the average amount of artificial sweetener consumption was $2 \mathrm{~g}$ (two packets) for an average duration of 5 years. Age, sex, family history of thyroid cancer in the firstdegree relative, and history of neck irradiation did not differ significantly between the two groups, as shown in Table 2. A proportion test showed a statistically significant number of artificial sweeteners consumers in group 1 than in group $2(76 \%$ vs. $24 \%, \mathrm{P}<0.05)$. An analysis done using binary logistic regression revealed that artificial sweetener consumption was significantly associated with increased risk of development of WDTC with an OR of 10.1 (95\% CI: $4.01-25.10, \mathrm{P}<0.05)$.

\section{Discussion}

The relationship between the consumption of artificial sweeteners and the development of malignancy in human beings is a complex subject for research owing to a broad range of both artificial sweeteners and tumors. Artificial sweeteners are now recognized as an ingredient of more than 6,000 food items of mass consumption, and many food products contain more than one artificial sweetener [9]. Mixing of two or more artificial sweeteners is done to inhibit the bitter after taste associated with some sweeteners, thereby making it difficult to study the association between single agents and specific types of cancer individually. These factors pose a significant challenge to researchers while conducting prospective trials determining the association between the use of artificial sweeteners and cancer. Most of the data regarding the carcinogenic potential of artificial sweeteners are derived from animals, and it must be born in mind that oncogenic mechanisms in experimental animals are known to differ from human beings [16, 17].

The controversy regarding artificial sweetener consumption and elevated risk of malignancy stems from an animal study in 1970 that showed an increased incidence of urothelial tumors in rats fed on cyclamate and saccharin $[1,18,19]$. Cyclohexylamine, a toxic metabolite of cyclamate, was implicated in the etiopathogenesis of bladder cancer [20-22], and further research revealed an increased risk of bladder tumors in rats fed on large doses of saccharin. Saccharin was removed from the list of potential cancer-inducing substances when the report of the National Institute for Environmental Health Sciences stated that cancer-causing pathways and mechanisms in rodents do not apply in human beings. Ascorbic acid (vitamin C) is also known to cause bladder carcinoma in rats when given at high doses similar to saccharin [16], and this can be prevented by administering prophylactic ammonium chloride to the rats. 
Table 2. Distribution of Selected Characteristics Among Thyroid Cancer Cases and Controls

\begin{tabular}{llll}
\hline & Differentiated thyroid carcinoma $(\mathbf{n}=\mathbf{5 0})$ & Benign thyroid nodule $(\mathbf{n}=\mathbf{5 0})$ & P value \\
\hline $\begin{array}{l}\text { Mean age } \\
\text { Sex }\end{array}$ & $52.4 \pm 11.09$ & $54.6 \pm 12.45$ & 0.648 \\
$\quad$ Male & 10 & 14 & 0.348 \\
$\quad$ Female & 40 & 36 & 0.439 \\
BMI & 16 & 11 & 30 \\
$\quad$ Underweight/normal & 24 & 9 & $<0.00001$ \\
$\quad$ Overweight & 10 & 12 & 2 packets \\
$\quad$ Obese & 36 & 1 & 0.307 \\
Artificial sweetener consumption & 4 packets & 6 & 0.504 \\
Average amount & 3 & 0 & 0.509 \\
Family history of thyroid cancer & 4 & & \\
Alcohol intake $\geq 2$ drinks/day & 1 & & \\
Irradiation in the childhood & & & \\
\hline
\end{tabular}

BMI: body mass index.

Rodents are particularly susceptible to developing urothelial tumors owing to their high urine osmolarity, which favors the precipitation of calcium phosphate crystals. The precipitated calcium phosphate crystals and high doses of many sodium salts are toxic to the superficial layer of the urinary bladder epithelium, thus causing regenerative hyperplasia and ultimately leading to tumorigenesis [16, 23]. Further research in monkeys $[24,25]$ showed that long-term intake of neither sodium saccharin nor sodium cyclamate was associated with increased susceptibility to the development of urothelial tumors. However, the data from these experiments have faced intense criticism owing to the small sample size of the study and the relatively lower dosages of saccharin used in the experiment.

In a study conducted by Olney et al [26] in 1996, consumption of aspartame was linked to increased incidence of brain tumors, and nitrosation of aspartame in the stomach was proposed to be the underlying mechanism [27]. Most of the epidemiological studies on human beings regarding artificial sweetener use relate to sweetener consumption in totality rather than to single agents specifically. The majority of data in humans is available in the form of case-control studies, and most of these studies published in the years 1965 - 1986 did not show an increased risk of bladder cancer with an intake of artificial sweeteners [7]. Sturgeon et al [8] conducted a casecontrol study where artificial sweetener use was classified as "low" (<1,680 mg per day) or "heavy" ( $>1,680 \mathrm{mg}$ per day) depending upon the amount of artificial sweetener intake. Heavy sweetener consumption ( $>1,680 \mathrm{mg}$ per day) was significantly associated with increased risk of bladder carcinoma (relative risk (RR): 1.3, 95\% CI: 0.9 - 2.1). Gurney et al in 1997 found that consumption of aspartame in children was not associated with the development of brain tumors [28].

There is a paucity of data on the effect of artificial sweeteners on the thyroid gland. Animal studies have linked the use of sucralose with alteration in the thyroidal axis leading to reduced levels of thyroid hormones along with decreased thyroid-stimulating hormone (TSH) [29]. Additionally, a study conducted on rats suggested that aspartame consumption can lead to biochemical picture resembling primary hypothyroidism. Formaldehyde (a metabolite of aspartame) was implicated to play a role in the etiopathogenesis of hypothyroidism by causing exhaustion of thyroid follicular cells [30]. Moreover, formaldehyde is also known to cause delayed type IV hypersensitivity reaction. Data from rodent models cannot be directly extrapolated to humans as thyroid neoplasm in rodents behave differently in certain aspects [17]. Thyroid neoplasms in rats are generally follicular in morphology, and the characteristic cytological features that are diagnostic for papillary thyroid cancer in human beings are usually absent. The current body of evidence suggests that rats are more sensitive than human beings to thyroid tumor induction due to hormonal disturbances that result in elevated TSH levels. The role of TSHstimulated growth in the etiopathogenesis of thyroid follicular neoplasms in rodents might be related to the increased likelihood of mutation that accompanies elevated mitotic activity or to the increased probability of expansion of clones of cells bearing pre-existing mutations [17].

A previous case report suggested the association between the autoimmune Hashimoto's thyroiditis with hypothyroidism and excessive consumption of beverages containing artificial sweeteners [31]. The patient recovered completely with normalization thyroid function tests and anti-thyroid peroxidase (TPO) antibody after discontinuation of artificial sweeteners. The patient remained clinically and biochemically euthyroid in the absence of any treatment during the subsequent follow-up visits. In animal studies, artificial sweeteners have also been linked to the development of the autoimmune disease. Alteration of gut microbiome induced by artificial sweeteners leading to dysregulation of the immune system was the proposed mechanism [32]. Researchers have shown that the use of sugar substitutes can decrease the total count of beneficial bacteria in the gastrointestinal tract, thus leading to increased gut $\mathrm{pH}$. 
This results in inhibition of the immune system and the thyroid function [33].

The past few decades have witnessed a dramatic increase in the consumption of artificial sweeteners. The incidence of differentiated thyroid carcinoma has also been increasing over the last decades and is mainly attributed to the increased detection rate of tumors at an earlier stage. Analysis of cases of WDTC (1988 - 2005) using the National Cancer Institute's Surveillance, Epidemiology, and End Results (SEER) dataset revealed an increase in the incidence of thyroid cancer for all sizes of tumors [11]. It was suggested that overdiagnosis is not only a single cause, and other factors, including dietary and environmental, could play a role. Zeng et al [34] conducted a retrospective case-control study to evaluate the relationship between biocide exposure and the development of thyroid cancer. Subjects whoever had a history of occupational exposure to biocides were found to have increased risk of thyroid carcinoma $(\mathrm{OR}=1.65,95 \% \mathrm{CI}: 1.16-2.35)$, and the maximum risk was seen for the higher cumulative odds of exposure (OR $=2.18,95 \%$ CI: $1.28-3.73$ ). Among all the thyroid cancers, microcarcinomas (tumor size $\leq 1 \mathrm{~cm}$ ) showed the strongest association.

Our study is the first to report a significant association between artificial sweetener consumption and well-differentiated thyroid carcinoma. The current study showed significantly increased intake of artificial sweeteners in patients with WDTC as compared to the control group. This study suggested that use of an average of four packets ( $4 \mathrm{~g}$ ) per day of artificial sweetener for an average duration of 5 years is associated with an increased risk of WDTC. The duration of artificial sweetener consumption did not differ significantly among the cases and controls. Patients with a diagnosis of WDTC consumed artificial sweeteners in higher dosages as compared to the control group. Our findings are in line with the earlier observation [8] that higher dosages of artificial sweeteners can have carcinogenic effects. Both groups did not differ significantly in terms of age, sex, family history of thyroid malignancy, and exposure to irradiation in childhood. Based on findings from our study, it is likely that increased consumption of artificial sweeteners could have contributed to the rise in cases of WDTC. The reason for the increased prevalence of artificial sweetener consumption in thyroid carcinoma patients is mostly unknown as we do not have any data in the literature regarding this association. Therefore, more extensive studies are needed in the future to elucidate the molecular pathways in detail, linking artificial sweetener use with the development of thyroid malignancy.

The possibility of inverse causality leading to bias is less likely because both the study groups had thyroid disease (one group with thyroid cancer and other group with benign thyroid nodule). Moreover, the history regarding duration and average amount of artificial sweetener consumption prior to the onset of thyroid disease was noted which virtually eliminated the modifying effect of disease on consumption of sweeteners. The present study is limited by its retrospective design, and subjects who have had the disease may search their memories more thoroughly than members of the control group for possible causes of their cancer. Moreover, the study relied on selfreported intakes of artificial sweetener and data were collected from only one interview or questionnaire. Hospital patients were used as controls, which could possibly skew the data. Nonetheless, these findings give some cause for concern. To address these limitations, prospective controlled study with a greater number of subjects is needed to determine the association between artificial sweeteners and thyroid cancer.

\section{Conclusions}

Although few studies are suggesting that a high dose, prolonged use of sweeteners might increase the risk of development of certain malignancies; the evidence for this is inconclusive. Our study is the first one to evaluate the relationship between sugar substitute use and the development of thyroid cancer. We found an increased risk of thyroid cancer with artificial sweetener consumption. This study emphasizes the significance of artificial sweetener consumption as a potential risk factor for WDTC and need to raise public awareness for this association. We also conclude that a positive association noted between artificial sweetener use and WDTC by themselves does not establish a causal relationship. Future evaluation of this relationship is warranted, and large-scale epidemiological studies determining the association between artificial sweetener consumption and thyroid carcinoma are necessary.

\section{Acknowledgments}

We appreciate the support of the Kozuki Foundation for Sports.

\section{Financial Disclosure}

None to declare.

\section{Conflict of Interest}

The authors declare that they have no conflict of interest.

\section{Informed Consent}

All subjects provided written informed consents.

\section{Author Contributions}

NS, SSL and IS has designed and performed the study. NS, SSL, SA and IS have drafted the manuscript and did critical editing. NS and SA performed statistics analysis. IS has carefully supervised and approved this manuscript.

\section{Data Availability}

The authors declare that data supporting the findings of this 
study are available within the article.

\section{References}

1. Bright G. Low-calorie sweeteners - from molecules to mass markets. World Rev Nutr Diet. 1999;85:3-9.

2. Weihrauch MR, Diehl V. Artificial sweeteners - do they bear a carcinogenic risk? Ann Oncol. 2004;15(10):14601465.

3. Helgren FJ, Lynch MJ, Kirchmeyer FJ. A taste panel study of the saccharin off-taste. J Am Pharm Assoc Am Pharm Assoc. 1955;44(6):353-355.

4. Moskowitz HR, Klarman L. The tastes of artificial sweeteners and their mixtures. Chem Senses Flav. 1975;1:411421.

5. Sylvetsky AC, Welsh JA, Brown RJ, Vos MB. Low-calorie sweetener consumption is increasing in the United States. Am J Clin Nutr. 2012;96(3):640-646.

6. Lindley MG. New developments in low-calorie sweeteners. World Rev Nutr Diet. 1999;85:44-51.

7. Morrison AS, Buring JE. Artificial sweeteners and cancer of the lower urinary tract. N Engl J Med. 1980;302(10):537-541.

8. Sturgeon SR, Hartge P, Silverman DT, Kantor AF, Linehan WM, Lynch C, Hoover RN. Associations between bladder cancer risk factors and tumor stage and grade at diagnosis. Epidemiology. 1994;5(2):218-225.

9. Lim U, Subar AF, Mouw T, Hartge P, Morton LM, Stolzenberg-Solomon R, Campbell D, et al. Consumption of aspartame-containing beverages and incidence of hematopoietic and brain malignancies. Cancer Epidemiol Biomarkers Prev. 2006;15(9):1654-1659.

10. Schernhammer ES, Bertrand KA, Birmann BM, Sampson L, Willett WC, Feskanich D. Consumption of artificial sweetener- and sugar-containing soda and risk of lymphoma and leukemia in men and women. Am J Clin Nutr. 2012;96(6):1419-1428.

11. Chen AY, Jemal A, Ward EM. Increasing incidence of differentiated thyroid cancer in the United States, 19882005. Cancer. 2009;115(16):3801-3807.

12. Howlader N, Noone AM, Krapcho M, Miller D, Brest A, Yu M, Ruhl J, et al. SEER Cancer Statistics Review, 1975-2017, National Cancer Institute. Bethesda, MD. https://seer.cancer.gov/csr/1975_2017/, based on November 2019 SEER data submission, posted to the SEER web site, April 2020.

13. Haugen BR. 2015 American thyroid association management guidelines for adult patients with thyroid nodules and differentiated thyroid cancer: what is new and what has changed? Cancer. 2017;123(3):372-381.

14. U.S. Food \& Drug Administration. May 19, 2014. Additional information about high-intensity sweeteners permitted for use in food in the United States. https:// www.fda.gov/food/food-additives-petitions/additionalinformation-about-high-intensity-sweeteners-permitteduse-food-united-states.

15. Franz M. Diet soft drinks: how safe are they? Diabetes Self Manag. 2010;27(2):8, 11-13.
16. Cohen SM, Anderson TA, de Oliveira LM, Arnold LL. Tumorigenicity of sodium ascorbate in male rats. Cancer Res. 1998;58(12):2557-2561.

17. Species differences in thyroid, kidney and urinary bladder carcinogenesis. Proceedings of a consensus conference. Lyon, France, 3-7 November 1997. IARC Sci Publ. 1999;147:1-225.

18. Wagner MW. Cyclamate acceptance. Science. 1970;168(3939):1605.

19. Price JM, Biava CG, Oser BL, Vogin EE, Steinfeld J, Ley HL. Bladder tumors in rats fed cyclohexylamine or high doses of a mixture of cyclamate and saccharin. Science. 1970;167(3921):1131-1132.

20. Renwick AG. The metabolism of intense sweeteners. Xenobiotica. 1986;16(10-11):1057-1071.

21. Bopp BA, Sonders RC, Kesterson JW. Toxicological aspects of cyclamate and cyclohexylamine. Crit Rev Toxicol. 1986;16(3):213-306.

22. Roberts A, Renwick AG. The pharmacokinetics and tissue concentrations of cyclohexylamine in rats and mice. Toxicol Appl Pharmacol. 1989;98(2):230-242.

23. Whysner J, Williams GM. Saccharin mechanistic data and risk assessment: urine composition, enhanced cell proliferation, and tumor promotion. Pharmacol Ther. 1996;71(1-2):225-252.

24. Takayama S, Renwick AG, Johansson SL, Thorgeirsson UP, Tsutsumi M, Dalgard DW, Sieber SM. Long-term toxicity and carcinogenicity study of cyclamate in nonhuman primates. Toxicol Sci. 2000;53(1):33-39.

25. Takayama S, Sieber SM, Adamson RH, Thorgeirsson UP, Dalgard DW, Arnold LL, Cano M, et al. Long-term feeding of sodium saccharin to nonhuman primates: implications for urinary tract cancer. J Natl Cancer Inst. 1998;90(1):19-25.

26. Olney JW, Farber NB, Spitznagel E, Robins LN. Increasing brain tumor rates: is there a link to aspartame? J Neuropathol Exp Neurol. 1996;55(11):1115-1123.

27. Shephard SE, Wakabayashi K, Nagao M. Mutagenic activity of peptides and the artificial sweetener aspartame after nitrosation. Food Chem Toxicol. 1993;31(5):323329.

28. Gurney JG, Pogoda JM, Holly EA, Hecht SS, PrestonMartin S. Aspartame consumption in relation to childhood brain tumor risk: results from a case-control study. J Natl Cancer Inst. 1997;89(14):1072-1074.

29. Palkowska-Gozdzik E, Bigos A, Rosolowska-Huszcz D. Type of sweet flavour carrier affects thyroid axis activity in male rats. Eur J Nutr. 2018;57(2):773-782.

30. Patel KG, Bhatt HV, Choudhury AR. Alteration in thyroid after formaldehyde (HCHO) treatment in rats. Ind Health. 2003;41(3):295-297.

31. Sachmechi I, Khalid A, Awan SI, Malik ZR, Sharifzadeh M. Autoimmune Thyroiditis with Hypothyroidism Induced by Sugar Substitutes. Cureus. 2018;10(9):e3268.

32. Mori K, Nakagawa Y, Ozaki H. Does the gut microbiota trigger Hashimoto's thyroiditis? Discov Med. 2012;14(78):321-326.

33. Schiffman SS, Rother KI. Sucralose, a synthetic organo- 
chlorine sweetener: overview of biological issues. J Toxicol Environ Health B Crit Rev. 2013;16(7):399-451.

34. Zeng F, Lerro C, Lavoue J, Huang H, Siemiatycki J, Zhao
$\mathrm{N}$, Ma S, et al. Occupational exposure to pesticides and other biocides and risk of thyroid cancer. Occup Environ Med. 2017;74(7):502-510. 